\title{
Cervical Alveolar Soft Part Sarcoma
}

National Cancer Institute

\section{Source}

National Cancer Institute. Cervical Alveolar Soft Part Sarcoma. NCI Thesaurus. Code C40225.

An alveolar soft part sarcoma arising from the cervix. 